\title{
Jeanne Hersch y la tradición filosófica*
}

\section{Jeanne Hersch and philosophical tradition}

\author{
Carmen Revilla GuZMÁN
}

Universidad de Barcelona

\section{Resumen}

El trabajo presenta una aproximación a la obra de Jeanne Hersch con el fin de reflexionar sobre el alcance de la influencia del pensamiento de Jaspers en ella. Como hipótesis se parte de la importancia que la autora concede a la tradición filosófica como forma de realizar una actividad teórica dirigida a la "comunicación" y articulada en torno a la dimensión poiética del ser humano. La identificación de algunas categorías nucleares en la filosofía herschiana, permite señalar algunos rasgos propios de su aportación, que caracterizan los distintos aspectos de su reflexión y se concretan en la atención al presente.

Palabras clave: Hersch, Jaspers, ontología, forma, tiempo, presente.

\begin{abstract}
This study reflects on the influence of Jasper's thought on the work of Jeanne Hersch. Our hypothesis is based on the importance that Hersch attributed to the

\footnotetext{
* Este trabajo se ha realizado en el marco del proyecto de investigación "Filósofas del siglo XX: aportaciones al pensamiento filosófico y político" FFI2009-08468, Ministerio de Ciencia e Innovación.
} 
philosophical tradition as a way of carrying out a theoretical activity aimed at "communication" and articulated around the poietic dimension of the human being. The identification of certain nuclear categories in Hersch's philosophy allows us to highlight certain distinctive features of her contribution, which characterize the different aspects of her reflection and materialize in the attention to the present.

Keywords: Hersch, Jaspers, ontology, form, time, present.

Raymond Klibansky, compañero de estudios de Jeanne Hersch en el círculo de Jaspers en Heidelberg, hablando de aquel entonces, escribe: "Había dos estudiantes que han llegado a ser bastante conocidas, una era Hannah Arendt y la otra, a mi juicio mucho más importante, Jeanne Hersch, filósofa de Ginebra. Las dos, y Jeanne Hersch sobre todo, en cierto modo, han continuado su pensamiento -y Jeanne Hersch tal vez lo ha superado también, permaneciéndole fiel"'. Esta observación, discutible sin duda y cuya valoración la propia autora no comparte ${ }^{2}$, sitúa, sin embargo, con bastante precisión el marco al que pertenece su aportación, una obra, considerablemente amplia y dispersa, que es, o ha comenzado a ser en la actualidad objeto de estudio e investigación detallada ${ }^{3}$. Su trabajo, por una parte, recoge la herencia jaspersiana y, por otra, se caracteriza por una expresa voluntad de encarnarse en un compromiso político y social, aspecto al que ella misma se refiere cuando reitera de sí misma que es "una presencia en su tiempo más que la autora de una obra"4; aunque quizá no sea posible hablar de formas de superación de unos autores respecto a otros, este doble aspecto de su reflexión es, sin duda, indicativo de la peculiaridad de su propuesta y de las posibilidades de desarrollo del planteamiento teórico que comparte con su maestro.

1 Klibansky, R.: Le philosophe et la mémoire du siècle, París, Les belles lettres, 1998, p. 25 ; cfr. Hersch, J.: Essere e forma, Prefazione de R. de Monticelli, Milán, Bruno Mondadori, 2006, p. IX. Roberta de Monticelli, una de las más autorizadas estudiosas de la obra de Jeanne Hersch, inicia su presentación de la traducción italiana de L'être et la forme con esta cita de Klibansky.

2 A Hannah Arendt explícitamente la considera autora de una obra mucho más importante que la suya; vid. Hersch, J.: Eclairer l'obscur. Entretiens avec Gabrielle et Alfred Dufour, Lausanne, L'Age d'Homme, 1986, p. 220.

3 En 1909 se ha fundado, en Zurich y con motivo de la celebración en 1910 del centenario de su nacimiento, la Société Jeanne Hersch. En su web puede encontrarse información biográfica y una completa bibliografía de y sobre la autora: http://www.jeanne.hersch-gesellschaft.ch/index.php?lang=fr

4 "Je suis plutôt une présence à mon temps que l'auteur d'une oeuvre". Cuando se le pregunta por el sentido de esta afirmación, responde, indicando cuál es su "estilo" de pensamiento: "Espontáneamente soy alguien que responde más que construir de forma autónoma. Reacciono a una situación, a una obra, necesito una suerte de provocación", en Eclairer l'obscur. Entretiens avec Gabrielle et Alfred Dufour, ed. cit, p. 53.

Esta expresión, da título al ensayo, de carácter introductorio, de E. Dufour Kowalki, Jeanne Hersch. Presence dans le temps, Lausanne, L'Age d'Homme, 199. 
En la línea de la filosofía de la existencia de Jaspers, Jeanne Hersch ha llevado a cabo un trabajo paciente de "esclarecimiento existencial", polarizado en la preocupación por la condición humana, que juzga esencial y constitutivamente paradójica, en la medida en que la entiende como centro que evidencia la sustancialidad problemática de la dimensión temporal; el núcleo de su investigación, en este sentido, lo constituye la relación entre la búsqueda de la verdad, que define la tarea filosófica, y la presencia en el tiempo, que proporciona el ámbito de inserción del pensar. La articulación e irreductibilidad de esta doble vertiente determina, a su juicio, la especificidad, justamente, de la condición humana: "concebir una verdad general y encontrarse enfrentado a una situación particular, viviendo su irreductibilidad" 5 , esto es, asumiendo, en un sentido marcadamente kantiano, que en ello radica la libertad. Su pensamiento es, pues, bajo este ángulo, también un pensamiento de la libertad, que se realiza, como Jaspers enseñaba, "en situación", un pensamiento que, por su parte, apunta a una suerte de fascinación por el instante, por el hic et nunc que hace del presente el lugar de la decisión. De hecho, ante el debilitamiento del sentimiento de la realidad que en buena medida es, a su modo de ver, uno de los rasgos de nuestra cultura, la autora aboga por "vivir del presente", algo para lo que resulta necesario "dejarse absorber por él [...], ser de algún modo como una esponja y absorber lo que se recibe"6.

A lo largo de su obra, Jeanne Hersch conjuga un sentido muy riguroso, y se diría que clásico, de la filosofía con la capacidad de asumir la actualidad y, sobre todo, con el empeño por responder a los requerimientos plurales del presente, diversificando de esta forma su intervención en el debate filosófico del siglo XX; su aportación, en consecuencia, invita a adoptar diferentes puntos de vista que permitan recoger la riqueza de sus reflexiones, sin abandonar la referencia a la unidad de sentido que preside y orienta su trabajo teórico.

Entre las perspectivas en las que la obra de esta autora podría ser abordada tal vez se puedan destacar como especialmente prometedoras: a) en primer lugar, la que atiende a las líneas principales en las que se desarrolla su tarea y da lugar a clasificar sus escritos bien en el ámbito de la filosofía práctica, subrayando la dimensión ética de la filosofía, bien en el de la estética, en virtud de las conexiones entre estética y ontología, así como sus preocupaciones pedagógicas, a partir de la importancia que concede al tema de la libertad; b) una segunda perspectiva es la que ofrece la consideración de su lugar en la tradición filosófica, en la medida en que permite no sólo percibir la relevancia en la génesis de su pensamiento de maestros e interlocutores, sino también el significado que adquiere en la elaboración del mismo la lectura de la historia, como ámbito al que ha dedicado especial atención; c) en tercer lugar, cabría tomar en consideración la particular relación de participación y

\footnotetext{
5 O. c., p. 168.

6 O. c., p. 223.
} 
distancia respecto a tradiciones y formas culturales que la autora misma reconoce como comunidad de pertenencia, entre las que resulta singularmente significativa su conexión, por ejemplo, con el pensamiento judío, aunque, en este sentido, adquiere una prioridad indiscutible la influencia de Jaspers. Cada una de estas perspectivas se abre a múltiples posibilidades de desarrollo, determinadas, en todo caso, por una inequívoca voluntad de intervención, de respuesta a lo que ella misma considera la "presión de las circunstancias"7.

Sosteniendo su amplia producción intelectual hay, sin embargo, lo que ella llamará un "gesto filosófico fundamental, escondido bajo las operaciones intelectuales"8: se trata del movimiento del pensar que un filósofo realiza en profundidad y que, en su caso, nace del convencimiento de que "toda reflexión filosófica compromete la existencia"9 en la medida en que pone en juego la libertad que define la condición humana; este movimiento, realización de la reflexión filosófica, radica en la presencia de un foco irreductible, capaz de entrar en relación con el fondo inagotable e inexpresable de lo real, al que el filósofo dirige su atención para hacerlo visible a través de pensamientos claros ${ }^{10}$; su interés por la esencia de lo particular remite a este origen que dota a su pensamiento de una profunda unidad y coherencia.

El modo en el que se dirige en sus trabajos de historia del pensamiento a los filósofos que considera depositarios de una perspectiva sobre lo real que permite un "ejercicio activo de razón y libertad"11 es, en este sentido, una expresión clara de lo que para ella es la función de la filosofía. Quizá por eso, en su obra, la tarea filosófica no es disociable tampoco de una constante y personalmente implicada actividad docente que será ocasión para llevar a cabo una importante reflexión de carácter pedagógico y, sobre todo, le proporciona el medio en el que poner a prueba la experiencia intelectual del trabajo teórico.

En sus objetivos y en la forma de entender la actividad filosófica es muy clara la influencia de Jaspers, al que siempre se referirá como su maestro, desde que escuchándole en Heidelberg ya en 1929, según su propio testimonio, descubrió "lo que era la filosofía"12. El reconocimiento explícito por parte de la autora de su deuda con Jaspers, así como la existencia de huellas evidentes de la misma, invita a cues-

\footnotetext{
7 O. c., p. 220.

8 O.c., p. 36.

9 Hersch, J.: L'étonnement philosophique. Une histoire de la philosophie, París, Gallimard, 1993, p. 388 (traducción al español de Rosa Rius Gatell de inminente aparición en Acantilado).

$10 \mathrm{El}$ comentario más preciso a esta función que la autora reconoce a la filosofía puede verse en Monticelli, R. de: "L'albero e la festa. Più che una postfazione, un omaggio" en Jeanne Hersch: La nascita di Eva. Saggi e racconti, Novara, Interlinea edizioni, 2000, pp. 75 ss., traducción al italiano de los ensayos publicados por Hersch como Textes en Friburgo, Le feu de nuict, 1985 (traducidos al español por Rosa Rius Gatell, con el título de El nacimiento de Eva, Barcelona, Acantilado, 2008).

11 Hersch, J.: L'étonnement philosophique, ed. cit., p. 455.

12 Hersch, J.: Eclairer l'obscur. Entretiens avec Gabrielle et Alfred Dufour, ed.cit., p. 25.
} 
tionar hasta dónde llega la fidelidad a su enseñanza con el fin de discernir ese margen de libertad en el que se inscribe su aportación; esta fidelidad, por otra parte, por debajo de cuanto entraña de actitud personal, parece presentar una conexión esencial con la relación que mantiene con la tradición filosófica, a la que accede en buena medida a través de este autor, aunque en ambos casos bajo el signo de una cierta ambigüedad: la vinculación a la propia experiencia y el empeño por hacer de su filosofar una respuesta personal al presente caracterizan el pensamiento herschiano simultáneamente por una inequívoca pertenencia a la tradición filosófica que convive con la apertura a otros territorios, por una lealtad que es también distancia, lugar y ocasión del ejercicio libre del pensar.

\section{Una cita con la historia}

En Eclairer l'obscur, a una pregunta sobre el modo en el que vivió acontecimientos como la guerra de España y la segunda guerra mundial, Jeanne Hersch respondía señalando la dificultad que le supone hablar de ello, porque, a pesar de su compromiso, "tengo la impresión -dice- de haber vivido este período casi ausente del mundo y enteramente devorada por mi vida personal, algo que no llego a perdonarme". Son los años en los que, tras la publicación de L'Illusion philosophique, prepara su tesis, escribe Temps alternés y diversos artículos, a la vez que se dedica a la enseñanza en la Ecole Internationale. Esta intensa actividad la lleva a afirmar que tiene "el sentimiento de haber faltado a mi cita con la historia, cuando era una joven adulta, perfectamente capaz de actuar", en otras palabras, de no haber conjugado satisfactoriamente "el lado personal de su vida, el petit côté de la historia" con "la gran historia humana" que, sin embargo, está esencialmente constituida por éste: "La gran historia y la historia personal, la yuxtaposición y el cruce de estos elementos, la manera en que se viven, jamás dejará de ser un problema para mí"13. En cierto modo, su biografía, presidida por este problema, viene a configurarse como una respuesta al mismo que se concreta en fórmulas singularmente elocuentes: "Espontáneamente soy alguien que responde, más que construir de forma autónoma", por ejemplo, o también: "Soy más una presencia a mi tiempo que el autor de una obra" 14 .

En efecto, la preocupación por el presente, su ocupación efectiva en el hic et nunc, no sólo está en el origen de los diversos planos de actividad que desarrolla, también se encuentra implicada en su concepción teórica de lo que es la filosofía, como lo evidenciaría el hecho de vincular, desde el inicio, su vocación filosófica a la enseñanza -"felizmente, no se me pide ser filósofo, sino solamente enseñar filo-

\footnotetext{
13 O.c., p. 37.

14 O.c., p. 53.
} 
sofía"15, afirma en la culminación de su carrera docente-, así como al reconocer en la filosofía misma una dimensión práctica que le es esencial: "La filosofía consiste esencialmente en ejercicios de libertad. Sólo que no se trata de cualquier ejercicio. Es de tal naturaleza que ejercer su libertad en este caso es al mismo tiempo atarse a una verdad única. Entonces uno se compromete a sí mismo en las operaciones mediante las cuales la busca"16. Ahora bien, entre estas operaciones parece reconocer un papel insustituible al descubrimiento "del gesto filosófico fundamental" que todo pensador realiza, un gesto, como recordaba antes, que permanece "escondido bajo las operaciones intelectuales" de las que se sirve, aunque sin agotarlo ni reducirse a ellas, y que hay que intentar "mimer", esto es, recrear. En esta implicación de dimensiones, que conforma el núcleo de la "comunicación" filosófica, parece apoyarse su interés por la actividad pedagógica, a cuya consideración dedica observaciones fundamentales, basadas en su propia dedicación docente y en las que se articula una idea de la filosofía que va forjando paulatinamente a partir de su experiencia y de algunas convicciones muy iniciales, que se remontan a su época de estudiante, cuando Jaspers le descubre la filosofía.

Entre estas convicciones iniciales ella misma ha reiterado la importancia de una que orientó la realización de su primer texto filosófico, la memoria de licenciatura sobre las imágenes en la obra de Bergson: "Entonces yo estaba ya convencida de que la forma en la que se expresa un filósofo es muy importante -mucho más tarde, en mi tesis, he escrito: «El fondo del fondo es la forma»"17. Es precisamente esta fórmula, de contenido ontológico preciso como veremos más adelante, la que me ha hecho pensar en la progresiva tendencia a la simplificación como indicio de algo que afecta al fondo de su pensamiento y viene a ser el esfuerzo logrado, y esencialmente volcado a la comunicación, que parece caracterizar su producción; este rasgo, adquirido ya desde sus años de formación intelectual18, es muy perceptible en textos manifiestamente de madurez, como lo es L'étonemment philosophique.

L'étonemment philosophique, publicado en 1981, es la versión francesa de Das philosophische Staunen que, a su vez, recoge un curso de filosofía para la Radio Suisse Romande, distribuido también, al parecer con una muy favorable acogida, a través de 40 casetes. La obra, una historia de la filosofía, en su opinión poco convencional, la concibe como una introducción al pensamiento filosófico y es, a mi

\footnotetext{
15 O.c., p. 23.

16 O.c., p. 36.

17 O.c., p. 33.

18 O.c., p. 20: recordando la enseñanza de su profesor de francés, François Bouchardy, que le enseñó lo que muy pocos enseñan: "el arte de corregirse", dice: "De él aprendí que si se quiere mejorar el estilo, ante todo hay que reducir el volumen de lo que se escribe. Una buena parte del arte de escribir consiste en tachar y abreviar. Esta lección me quedó para siempre. Más tarde, cuando he colaborado en obras colectivas, he constatado con un cierto orgullo que, entre todas las contribuciones, la mía en general era la más corta”.
} 
modo de ver, una intervención ejemplar de "alguien que responde" y acude así a su "cita con la historia". A través de un extraordinario esfuerzo de síntesis y clarificación elabora aquí, guiada por la intención de "comunicar", una respuesta personal a lo que a su juicio son las necesidades de una cultura, la nuestra, depositaria, como toda cultura, de las virtualidades de la libertad ${ }^{19}$, que ahora ve amenazadas. Y aquí, en esta obra, adquiere esta respuesta una formulación que, bajo la sencillez que ella misma llega a calificar, sin duda impropiamente, de "trivial", unifica su vocación estrictamente filosófica con la idea ya muy madurada de la condición humana que ha centrado su reflexión.

Para Jeanne Hersch el ser humano es "algo excepcional" y "único" en su condición de "testigo del mundo", función que realiza por medio de "la visión, la contemplación y el conocimiento" 20 . Con la elección del título de la obra subraya este aspecto y nos indica, desde la "Advertencia" inicial, que "el asombro es esencial a la condición humana": "saber asombrarse es lo propio del hombre"; el asombro describe la existencia que, desde una actitud que concierne a la vida del sentir, se cumple y opera dando "forma"; por ello, lo que pretende es "suscitar de nuevo este asombro" que, en la historia, ha dado lugar al surgimiento de una pluralidad de miradas y formas de situarse en el mundo que son otras tantas formas "de ser un ser humano" 21 . Lejos de responder, pues, a una mera curiosidad cultural, su proyecto lo hace a la necesidad perentoria que nuestro tiempo tiene de "pensar filosóficamente". Como ella misma ha explicado, esta necesidad encuentra su raíz actualmente en la creciente aceleración de los cambios a los que asistimos, con el consiguiente y continuo requerimiento de una toma de posición ante situaciones nuevas, que crean una incertidumbre generalizada, origen de distintas formas de violencia, que van del dogmatismo y la crispación al ejercicio de la fuerza, y que son expresión de una "profunda inseguridad"; en esta situación se inscribe, como un factor muy determinante, el sorprendente conformismo suscitado y afianzado por los medios de comunicación que tienden a imponer una opinión dominante a través de consignas y clichés. Partiendo de este diagnóstico de la actualidad la autora se propone revitalizar en nuestras sociedades la reflexión filosófica, como ejercicio de libertad y pluralismo, capaz de proporcionar certezas vitales y garantía de una existencia propiamente humana. Su recorrido por la historia de la filosofía occidental pretende, pues, proporcionar los medios para llevar a cabo una reflexión filosófica, incluso a quienes no tienen esta formación, desde el convencimiento de que el trato con esta tradición, en esta perspectiva, nos permitirá, en primer lugar, "ejercitar diversas maneras de ser hombre", esto es, "de pensar, de situarse en relación al mundo, y de creer", en segundo, proporcionará "un utillaje de pensamiento, un cierto vocabulario, y, con el

\footnotetext{
19 O.c., p. 183.

20 O.c., p. 59.

21 O.c., p. 181.
} 
vocabulario, un cierto número de nociones, que permitirían plantear de manera más adecuada los problemas contemporáneos"; $y$, aunque sólo en tercer lugar, alude al objetivo de "alumbrar eventualmente una curiosidad en profundidad, a la espera de que alguien quizá se una a la reflexión filosófica" 22 .

La precisa determinación de sus objetivos, la perspectiva en la que lleva a cabo la selección de los autores de los que se ocupa y la claridad de su exposición son elementos característicos de esta obra, profundamente trabados entre sí y con aspectos básicos y fundamentales de su posición teórica. Por eso, más que en el comentario detallado de cada una de las presentaciones de figuras y épocas, quisiera detenerme brevemente en los rasgos generales de su acercamiento a la historia del pensamiento, con el fin de matizar su ubicación en la tradición filosófica y su posible relación con el modo en el que se fragua su propio filosofar. En este sentido tal vez se pueda aventurar una doble hipótesis: en primer lugar, que su idea de lo que es la filosofía, de impronta claramente kantiana y jaspersiana, se gesta en buena medida en el trato con esta tradición del que esta obra deja constancia de forma modélica, $\mathrm{y}$, por otra parte, que la contribución efectiva de este texto al desarrollo de su reflexión sobre la condición humana, así como su aportación al "aquí y ahora", se cifra, también ejemplarmente, en el modo de expresión, esto es, en la forma que aquí imprime a su pensamiento, concretada en las características que señalaba y dotada de una considerable eficacia.

Uno de los primeros rasgos que llama la atención en esta singular y poco "tradicional" historia de la filosofía es el hecho de atender preferentemente a figuras, los pensadores que considera "más típicos, los que ofrecen un modelo de toma de posición fundamental respecto al mundo" 23 , más que a la caracterización de épocas o movimientos filosóficos, a los que se refiere sólo ocasionalmente y por motivos muy precisos y relevantes desde el planteamiento del que parte. En este sentido se refiere a los epicúreos y los estoicos, como escuelas que proporcionan una ayuda a la vida práctica, aunque la dimensión práctica de la reflexión filosófica aparece tratada con detenimiento ya al presentar a Sócrates y Platón; también alude, en general, a la Filosofía Medieval por su aportación a la precisión y profundidad del lenguaje filosófico, o al Renacimiento en virtud de que sus características "hacen pensar en nuestro tiempo", que podría encontrar en sus elaboraciones ejemplos de lo que fue la conquista del "derecho a imaginar", de su capacidad para hallar principios de vitalidad en las fuentes, en la naturaleza y en la vida, así como ofrecernos quizá las primeras formas de organización del trabajo colectivo en el tiempo y, naturalmente, la revolución científica, de modo que su interés por esta época lo es por comprendernos y comprender de dónde venimos ${ }^{24}$. Por otra parte, el capítulo sobre

22 O.c., pp. 181-182.

23 Ibid.

24 O.c., p. 132. 
"El empirismo inglés" aborda, en concreto, la obra de Locke, Berkeley y Hume, si bien sólo muy parcialmente y, como explícitamente reconoce, con el fin de destacar su incidencia en Kant, esto es su aportación al tema de los límites del conocimiento y a la formación de la actitud crítica. También de carácter aparentemente general es el capítulo "De Kant al idealismo alemán", dedicado a subrayar que la filosofía kantiana es "la filosofía misma", una filosofía que supera los planteamientos ilustrados y que ella, por su reconocimiento del límite de lo fenoménico, no entiende como idealista25. Las páginas sobre lo que acontece "Después de Kierkegaard y Nietzsche" tampoco son la presentación de un movimiento filosófico, sino la explicitación de las consecuencias de la aportación de estos autores a la contemporaneidad, esto es, del compromiso de la filosofía con la existencia y la afirmación de la libertad como condición de la filosofía misma ${ }^{26}$. Igualmente el volumen se cierra con una reflexión sobre "La filosofía hoy" de carácter más bien un carácter conclusivo.

En último término, la dimensión práctica de la filosofía, su valoración del lenguaje filosófico, la importancia de la lectura del pasado, la incidencia de la ciencia y los márgenes del diálogo con ésta, la centralidad de Kant, "el filósofo por excelencia" que supo situar al ser humano en los límites de su condición, el esfuerzo por determinar la contemporaneidad, por ejemplo, son algunos de los hilos conductores que va así introduciendo y son decisivos en su propio planteamiento. Su preferencia, sin embargo, por centrar la exposición en torno a la obra de determinados filósofos, así como la selección de los mismos, renunciando a poner el acento en la continuidad, obedece a lo que es su objetivo: hacer que el lector reencuentre "su capacidad de asombro en el asombro de otros", presentar el asombro de los filósofos como origen de un "proceso creador" de modo que el lector se vea conducido a "filosofar él mismo", transmitiéndole los medios que le permitan su expresión27, porque, como dirá, los grandes filósofos son para los posteriores, y por extensión para todos y también para nosotros, "medios de expresión" e "instrumentos que utiliza su propio pensamiento", hasta el punto de reconocer la posible fecundidad incluso del malentendido 28 .

A este objetivo se dirige el procedimiento del "mimer", momento inicial de la comunicación filosófica y requisito para la comprensión de un texto, que consiste en rehacer el filosofar de un autor recreándolo. De este procedimiento la exposición de Jeanne Hersch proporciona un ejemplo claro, al identificar en el asombro de los filósofos que aborda el "gesto filosófico" que origina y dota de coherencia a su pensamiento. A lo largo de la obra, por otra parte, encontramos referencias explícitas a

\footnotetext{
25 O.c., pp. 253-257.

26 O.c., p. $388-391$.

27 O.c., pp. 7-8.

28 O.c., p. 61.
} 
esta práctica: a la importancia que tuvo en san Agustín el haber "mimé" el trayecto del pensamiento platónico 29 , o al enriquecimiento que supone su ejercicio con determinados autores, como Bergson 30 ; encontramos también indicaciones sobre textos concretos -sobre la Monadología, por ejemplo, de la que, a diferencia de lo que sucede con otras obras, "hay que comprender cada párrafo, cada línea, cada palabra" $31_{-}$, o sobre autores que exigen "acostumbrarse a su estilo" 32 , como sucede con la "expresión indirecta" kierkegaardiana; y encontramos, por supuesto, indicaciones en torno Kant, de cuya "capacidad de poner al desnudo las condiciones fundamentales de la condición humana" nadie "comprometido en la filosofía" puede escapar: su obra, nos dice, no puede ser fragmentada si quiere ser comprendida, porque "nos pide sumergirnos en profundidad en su reflexión más abstracta hasta alcanzar, a través de ella, "en la mimant", la situación concreta de un hombre, en la actualidad del mundo"33; introduce así el tema de la "comunicación", que aparecerá en la exposición de la filosofía de Jaspers.

Otra de las notas recurrentes en L'étonnement philosophique que también sorprende es la frecuente relación que establece de forma explícita entre los problemas y planteamientos que trata y la actualidad, o al menos con autores mucho más próximos cronológicamente y a ella, sin duda, más cercanos. Se trata de aproximaciones, a veces muy concretas, que, aunque sólo en ocasiones confirman la existencia de una tradición como tal, en todo caso salvan la continuidad de la exposición y resultan muy clarificadoras, además de acercar el pasado filosófico a los problemas del presente en la medida en que, al traducirlo a otros términos, favorecen su comprensión y ponen en juego el "débat", del que el "mimer" sería el preludio34. Se podrían multiplicar los ejemplos: la presentación de las aporías de Zenón acudiendo a la explicación bergsoniana de cómo y por qué pensamos el tiempo con ayuda de la inmovilidad ${ }^{35}$, el rechazo de J. Wahl al sistema en la exposición de Aristóteles ${ }^{36}$, las distancias entre éste, o Kant, y Hegel ${ }^{37}$, la lectura crítica que Simone Weil realiza de Marx $^{38}$ o la que hace Jaspers de Freud ${ }^{39}$, las referencias al "mito" de Nietzsche ${ }^{40}$, o a nuestra dificultad para "mimer" hoy el argumento onto-

\footnotetext{
29 O.c., p. 89.

30 O.c., p. 342.

31 O.c., p. 166.

32 O.c., p. 361.

33 O.c., p. 249.

34 O.c., p. 455.

35 O.c., p. 24.

36 O.c., p. 52.

37 O.c., pp. 265-267.

38 O.c., p. 301.

39 O.c., pp. 322-325.

40 O.c., pp. 385-387.
} 
lógico de san Anselmo41 o la experiencia intelectual de la evidencia tal como Descartes la vivió y somos incapaces de revivir 42 ..., por señalar sólo algunos casos. A éstos habría que unir, quizás ahora, la inclusión de Husserl, que "no es su filósofo", en función de la relevancia inesquivable que le reconoce en la configuración del pensamiento contemporáneo.

Tal vez precisamente el ejemplo de Husserl, cuya obra, a pesar de sus reticencias porque no le ha sido posible "pensar con él" 43 , presenta con una claridad y precisión análogas a las que encontramos en la presentación de otros autores, nos permita poner de relieve hasta qué punto el procedimiento de lectura que la autora pone a prueba resulta realmente eficaz. Por supuesto, la valoración del grado en el que los objetivos comunicativos de la autora se cumplen remite a los lectores; por eso, no deja de causar sorpresa cuando, al finalizar una exposición de la aportación husserliana, concisa pero en muchos sentidos modélica, nos interpela diciendo: "El lector se habrá dado cuenta sin duda del hecho de que al exponer la doctrina de Husserl no he conseguido alcanzar el mismo grado de claridad que con otros filósofos [...] He hecho lo que he podido. Pero quiero poner en guardia al lector: no se comprende a un filósofo - como ya he indicado- sino cuando se ha conseguido pensar con él. Aquí, esto no me ha sido posible"; aunque tampoco le ha sido posible, nos dice, dejarlo de lado, ya que "su influencia en la filosofía contemporánea, como también en las diversas ciencias humanas es demasiado evidente y demasiado profunda" 44 .

Jeanne Hersch denuncia las oscilaciones teóricas y la artificiosidad e imprecisión de vocabulario de Husserl, así como el hecho de que oscurezca lo que pretende superar o el que su presencia en la cultura contemporánea sea tan general como difusa, de forma que es su misma ambigüedad, nos dice, lo que parece haber multiplicado su influencia; aunque las páginas que le dedica no son sólo un paréntesis en L'étonnement philosophique, ni una concesión en reconocimiento a la importancia de su aportación, la insalvable falta de claridad que en él acusa le impide esa recreación, el mimer, previa e imprescindible al débat en el que se cumple la "comunicación" filosófica y que encontraría en el trato que mantiene con Jaspers un contrapunto modélico.

L'étonnement philosophique es una obra de considerable madurez en la que Jeanne Hersch va trenzando la exposición de su propia formación en el trato con la tradición filosófica con la presentación, muy sintetizada y orientada a unos objetivos precisos, de algunos hitos fundamentales que selecciona -y esta selección es muy sintomática- desde una concepción de la filosofía y el filosofar que ha ido fraguando justamente a través de su propio recorrido. A lo largo de la lectura del texto

\footnotetext{
41 O.c., p. 110.

42 O.c., p. 136.

43 O.c., p. 406.

44 Ibid.
} 
adquirimos no sólo información sobre los autores que trata, sobre su asombro, sus problemas y su vocabulario; a través de éstos, y como consecuencia de la forma en la que nos los presenta, se perfila también con nitidez el compromiso personal de la autora con su vocación filosófica.

\section{La ontología de las obras humanas}

La lectura de esta obra, L'étonnement philosophique, nos pone ante algunos grandes motivos, que, en el texto, aparecen ya en su lectura de los filósofos griegos, que se mantienen como constantes articuladoras y que, sin ser ajenos a la enseñanza de Jaspers, caracterizan su proyecto intelectual: a) la preocupación por la identidad de lo filosófico, su relación con la ciencia y su apertura a la interdisciplinariedad; b) la reflexión filosófica como práctica de autotransformación y su sustancial dimensión pedagógica; c) la valoración del principio sistemático y de la forma. A estos motivos habría que unir el alcance que adquiere su profunda relectura de la filosofía moderna, centrada en torno a Kant, como referencia determinante en su "meditación racional en los límites de la condición humana" 45 y en la que se sustenta su reflexión sobre la contemporaneidad en torno a los problemas del límite, el tiempo y la libertad.

En estos motivos se perfila, sosteniendo el andamiaje teórico de la obra y como líneas maestras, ese modo de entender la filosofía que ella misma nos dice haber descubierto en sus años de estudiante al escuchar a Jaspers y lo que podría considerarse específico de su aportación: la atención a la dimensión poiética de la condición humana, aceptando la "densidad de la materia" como principio de limitación.

En el proyecto de "esclarecimiento existencial" herschiano, animado por su arraigado "espíritu de situación y sentimiento de los límites", es decisivo también el reconocimiento de lo que en los seres humanos es irreductible al mundo de la causalidad material; se trata de un proyecto de clara inspiración en Jaspers y Kant, como ella misma reconoce, pero que adquiere una fisonomía propia al centrarse en el análisis de la acción conformadora que define simultáneamente la condición humana y la determinación de su mundo, una acción en la que se afronta la prueba de la realidad participando en la creación, y que encuentra su expresión paradigmática en el ámbito de la estética. Al análisis, en cierto modo sistemático, de este tema Jeanne Hersch dedica El ser y la forma, una obra de 1946, concebida como ontología de las obras humanas y de la que se ha dicho que, de alguna manera, cierra el ciclo de las grandes construcciones ontológicas de la primera mitad del siglo $\mathrm{XX}^{46}$.

45 O.c., p. 436, aunque ésta es la definición de la Filosofía en Jaspers.

$46 \mathrm{La}$ obra se inscribe en el ciclo representado, por ejemplo, por las grandes elaboraciones de: Heidegger: Ser y tiempo (1927), Jaspers: Filosofia (1931), E. Stein: Ser finito y ser eterno (redactado entre 1930 y 1940, aunque publicado póstumo en 1951), Sartre: El ser y la nada (1943). 
En esta obra, de contenido fuertemente teórico y de factura casi excesivamente clásica, aparecen esos rasgos de su pensamiento y de su producción que sus lectores han destacado como especialmente característicos: la confianza en la razón y el recelo de cuanto tiende a minarla en muchas de las propuestas de sus contemporáneos, el fondo de realismo que se concreta en el respeto a lo existente, el mantenimiento, en fin, de un ideal de claridad frente a la "palabrería" que ofusca o a la introducción de una "jerga" que paraliza la reflexión 47 , son algunos de estos rasgos, que, por ejemplo, Czeslaw Milosz señala explícitamente cuando se le pregunta qué ha aprendido de Jeanne Hersch 48 .

A partir de la consideración de las "modalidades de la forma" en los campos del conocimiento, la contemplación, la acción y el arte, El ser y la forma recoge una reflexión sobre el hacer propiamente humano, efectuación específica de su relación con el ser. La noción de "forma", de matriz kantiana, se convierte así en la categoría nuclear de la ontología que la autora elabora. Es ésta una categoría que explicita aquella convicción inicial que presidió sus primeros trabajos, según la cual "el fondo del fondo es la forma", y lo hace dotándola de un alcance que sustrae a esta fórmula del terreno "formal", entendido como meramente literario o retórico, o, mejor, reconduciéndolo al plano en el que adquiere su sentido esencial.

El ser y la forma parte de un claro planteamiento crítico, explícito desde la "Introducción" y en el que es muy clara la coincidencia con el modo en el que Jaspers presenta su tarea de elucidación del kantismo ${ }^{49}$, aunque también lo será, en su desarrollo, la diferencia de acentos en distintos temas; para ambos autores, la filosofía de Kant es una filosofía de la conciencia en la que la razón, "precisamente en cuanto razón, sabe de la finitud del hombre y experimenta sus propios límites" ${ }^{50}$, de tal modo que la finitud conduce a la "irremediable escisión" entre el acaecer natural y la libertad51. Ahora bien, si Jaspers se centra en su análisis en la relación sujeto-objeto, interesado por el posible origen común de sensibilidad y entendimiento, Hersch, por su parte, parece dejar a un lado estas cuestiones para centrar su atención en discernir netamente el nivel de la experiencia, de la pluralidad cambiante y heterogénea, como nivel de realidad en el que el ser puede ser nombrado, de aquel otro, que correspondería a la noción de ser cuya esencia implica la existencia y que juzga innominable. De este planteamiento se hace eco Roberta de

\footnotetext{
47 A la consideración del vocabulario filosófico en este sentido dedica unas interesantes observaciones en torno a lo que llama "términos abstractos intencionales", esto es, palabras vacías de sentido actual, cuyo equivalente ontológico se convierte en un "ídolo intelectual”, vid. Hersch, J.: El ser y la forma, trad. Adolfo Alfredo Nogrotto, Paidós, Buenos Aires, 1969, pp. 95-96.

48 Vid., por ejemplo, http://www.uuchristian.net/prophetmotive/archives/000208.html

49 Jaspers, K.: Los grandes filósofos, p. 224.

50 O.c., p. 321.

51 O.c., p. 322.
} 
Monticelli cuando, en el "Prefacio" a la traducción italiana de la obra, subraya que se trata de una "suerte de ontología negativa" en la medida en que asume como consigna el imperativo, dice recogiendo una expresión herschiana, de "no tomar el nombre del ser en vano" 52 , distanciándose así del núcleo problemático que tan poderosamente atraería el pensar heideggeriano. Consciente del alcance de la "diferencia ontológica" 53 , que entiende como consecuencia de un inevitable "antropomorfismo", sitúa su reflexión en el plano de lo óntico; es éste el plano de la acción que consiste en dar forma a cuanto nos circunda, aunque no deja de reconocer la presencia de un trasfondo inaccesible e innombrable, que, sin embargo, ha de quedar como tal. En este plano, su objetivo será elaborar una "teoría de la forma", partiendo de que "nada tiene realidad en una conciencia humana si no ha tomado forma", por eso, dirá, "explorar el antropomorfismo de la conciencia humana significa estudiar las condiciones necesarias para que algo sea real en la conciencia [...] empresa crítica de la que Kant es, una vez más, el precursor"54.

La noción de "forma", que constituye el eje de esta obra, designa el ser especificado y limitado, en definitiva, "todo lo que existe en la conciencia" 55 , como resultado de la "captación" -que descristaliza el mundo e introduce la diversidad56- de una materia por parte del espíritu. En virtud de su universalidad, la imposición de una captación a lo que nos es dado define también la condición humana ${ }^{57}$ : "el yo no es el yo sino por su acción transitiva" sobre el no-yo, que, a su vez, "no es real sino por el yo que se inserta en él", adquiriendo así diferentes "modos de existencia"58 que registran la impronta humana sobre la materia: "Toda actividad humana implica la captación de una materia para darle forma. Toda impronta del hombre en una materia presenta los caracteres de la forma [...] Sólo es accesible para el hombre lo que lleva ya el rastro de su captación. Se sigue de ello que la realidad para el hombre está enteramente en el dominio de la forma. La forma es para él el modo de cualquier realidad, de lo real como tal. Esto significa que lo que no tiene forma no puede ser para el hombre una experiencia sensible, ni una experiencia afectiva, ni una experiencia práctica, ni una experiencia intelectual, ni una experiencia estética, ni

\footnotetext{
52 Monticelli, R. de: "Prefazione" a Hersch, J., Essere e forma, trad. al italiano de S. Tarantino y R. Guccinelli, Milán, Bruno Mondadori, 2006, pp. XII ss.

53 "No es acerca del pensamiento y de sus condiciones que quisiéramos saber y decir algo; es acerca del ser. Si no podemos decir cómo es, quisiéramos por lo menos afirmar que es. Sin embargo, con esa sola afirmación lo hacemos descender del rango de el ser, al de un ser”, Hersch, J.: El ser y la forma, ed. cit., p. 21.

54 O.c., p. 22.

55 Ibid.

56 O.c., p. 25.

57 O.c., p. 42.

58 O.c., p. 29.
} 
nada. Lo que no tiene forma no podría ser percibido, ni concebido. La forma se extiende pues sobre toda la realidad humana" 59 .

En el análisis que la autora lleva a cabo es especialmente importante la atribución a la noción de "forma" de dos caracteres que le son esenciales: a) reconociendo, en este sentido, la herencia aristotélica además de kantiana ${ }^{60}$, la "forma" herschiana es una noción "dinámica" que designa "lo que sucede": la forma es un suceso y el rastro que deja61; b) por otra parte, y en virtud de la movilidad del límite entre captación y materia, la forma entraña una "invencible ambigüedad" en su demarcación respecto a la materia; esta ambigüedad, que es el signo de la libertad62, determina la especificidad de la condición humana y da lugar a que esta noción sólo tenga significación actual aplicada a las realizaciones humanas 63 .

En el mundo, esencialmente humano, de las formas "es necesario aceptar las escalas limitadas" 64 , porque es el mundo de la individuación plural y de la finitud; en él "la forma no tiene el mismo valor modal para todas las actitudes humanas, es decir, la misma perfección de ser o la misma relación con el ser"65; para la actitud teórica, en el conocimiento o en la contemplación, y para la actitud práctica, ética o política, la forma, provisional y con carácter de medio, apunta a algo más allá de la condición humana, al ámbito de lo innominable66. Sólo en un plano de la existencia encuentra la forma "su realización definitiva": el arte 67 . La concepción dinámica de las relaciones entre materia y forma, esto es, el principio que guía su planteamiento ontológico, encuentra en la obra de arte la expresión paradigmática de la condición humana y modelo de lo que el ser humano puede hacer existir; por eso, dirá, "el estudio de la forma debe hacerse sobre todo en el dominio del arte", porque "es en él, sólo en él, donde las formas creadas subsisten por sí mismas y encuentran su razón de ser en su sola existencia. Fuera de él, en otros modos de actualización humana, ellas deben permitir crear formas nuevas que las reemplazarán, y subordinarse así a un valor que las trasciende. Pero el arte no tiene otro fin que el de crear formas, formas definitivas" 68 . El pensamiento herschiano se ve así inmerso en el ámbito, específico y dotado de una legalidad propia, de la estética, ámbito al que concederá una atención preferente no sólo en esta obra.

\footnotetext{
59 O.c., p. 93.

60 "Creo que no se entiende a Kant cuando se lo lee renunciando totalmente al dinamismo insuflado por Aristóteles a la noción de forma", o.c., p. 57.

61 O.c., pp. 88 y 95.

62 O.c., pp. 55 ss.

63 O.c., p. 100.

64 O.c., p. 146.

65 O.c., p. 118.

66 Ibid.

67 O.c., pp. 120-121.

68 O.c., p. 147.
} 
Si el modo en el que Jeanne Hersch desarrolla su teoría de la forma le permite poner el acento, sin abandonar el terreno de la ontología, en sus implicaciones interdisciplinares y de orden práctico, la relevancia que adquiere el análisis de la cuestión estética resulta decisiva para la comprensión y valoración del alcance de su propuesta, en la medida en que explicita lo que ella misma considera rasgo definitorio de su postura: un "realismo fundamental" que "se manifiesta en la importancia concedida al cuerpo y a la realidad objetiva material" 69 .

Al llegar a este punto, que orienta el rumbo de las investigaciones de la autora, y tras haber recorrido algunas páginas importantes de su obra, es fácil reconocer en ellas la impronta inequívoca de aspectos de la filosofía de Jaspers ${ }^{70}$, tal como él mismo incluso los ha expuesto sintéticamente, por ejemplo, en su Autobiografia filosófica: la búsqueda de claridad conceptual y precisión en la definición de nociones, patente desde que iniciara sus estudios de psiquiatría 71 , la preocupación gnoseológica y metodológica que, incentivada por la fenomenología, impulsó ya la elaboración de su Psicopatología general ${ }^{72}$, el convencimiento de que la filosofía no es ciencia, pero tampoco se desarrolla en la "arbitrariedad del sentir", de que la "verdad filosófica" "ningún enunciado puede captarla adecuadamente" por lo que "sólo se presta a la comunicación indirecta"73, convencimiento que se encuentra en la base de la Lógica filosófica dirigida a determinar las condiciones de la comunicación 74 , la consideración de Platón y Kant como "cúspides" de la Filosofía 75 ... Sobre el fondo de una sintonía de planteamiento entre los dos autores se inscriben algunos de los tópicos de la filosofía existencial de Jaspers, cuya génesis nos presenta en el relato de su trayecto y que Hersch, sin duda, comparte: la "fe filosófica" jaspersiana, esto es, "la fe en el camino de la verdad, en el que cabe el encuentro de todos los que la buscan con sincero empeño"76, determina también su actitud teórica, así como es compartida por ambos la conciencia de la inacababilidad de su tarea: "jamás he llegado al final" 77 , confiesa Jaspers, mientras que Jeanne Hersch se presenta como un "océano de puntos de interrogación"78.

Todos estos motivos, entre otros, dibujan un marco teórico en el que la afinidad entre los dos autores es manifiesta. Y sin embargo, también lo es la diferencia en un

${ }^{69}$ Hersch, J.: Eclairer l'obscur, ed. cit., p. 203.

70 Entre los múltiples trabajos en los que Hersch trata aspectos de la filosofía jaspersiana, destaca: Kart Jaspers, Lausanne, L'Age d'homme, 1978.

71 Jaspers, K.: Autobiografía filosófica, trad. Pablo Simón, Buenos Aires, Sur, 1964, p. 21.

72 O.c., p. 22.

73 O.c., p. 81 .

74 O.c., p. 84.

75 O.c., p. 92.

76 O.c., p. 94.

77 O.c., p. 99.

${ }^{78}$ Hersch, J.: Eclairer l'obscur, ed. cit., p. 201. 
punto, que el propio Jaspers destaca como básico: "Desde siempre había sido objeto de mis afanes discernir lo verdaderamente grande, lo imprescindible y esencial", una "inclinación a la grandeza" progresivamente intensificada que le llevó a interesarse por la experiencia de las "situaciones límite" en las que el ser humano cobra conciencia de su propia esencia y a fijar su atención en la "humanidad en su conjunto"79. Frente a ello, la capacidad de volcarse en el presente, la sensibilidad a la "presión de las circunstancias" que Hersch incorpora a su "gesto filosófico" modula su pensamiento con rasgos específicos, que ella califica de "debilidad creadora" y reconoce en su preocupación por el "hacer" y en la dispersión de su vida y de su obra; por debajo de esta supuesta "debilidad" y "dispersión" discurre ese empeño continuo en cultivar el "sentido para lo que tiene sentido" 80 en el que radica su "presencia en el tiempo", y que es la de un centro vivo y responsable: "En la orquesta del mundo he sido un pequeño instrumento con timbre propio" 81 .

La teoría de la forma, anclada en la tradición pero reconducida a la explicitación de la condición humana, que la autora elaboró en 1946 le permite forjar la noción a través de la cual encauzará el tratamiento de muchos de sus temas más característicos, por lo que tiene, por una parte, de revalorización de la receptividad y de "realismo fundamental" que abre su reflexión al reconocimiento de la corporeidad y de la finitud, por otra, de poiesis, esencial al proceso de recreación del mimer, que sintoniza así con la acción formativa llevada a cabo en la creación estética, tal como se analiza, especialmente, en El ser y la forma. En esta obra, pues, se esbozan categorías nucleares de la filosofía herschiana y de su "gesto filosófico", en las que se percibe su "timbre propio" y el modo en el que acude a su "cita con la historia".

Carmen Revilla Guzmán

Departamento de Historia de la Filosofía, Estética y Filosofía de la Cultura

Facultad de Filosofía

Universidad de Barcelona

crevilla@ub.edu

\footnotetext{
79 Jaspers, K., Autobiografía filosófica, ed.cit., pp. 95-98.

80 Hersch, J.: Eclairer l'obscur, ed. cit., p. 167.

81 O.c., p. 225.
} 